\title{
VITAMIN B12 DEFICIENCY IN TYPE I DIABETES
}

\author{
Sana Ashiq \\ Institute of Microbiology and Molecular Genetics, University of the Punjab, Quaid-e-Azam Campus, Lahore-54590, Pakistan \\ Shabana* \\ Institute of Microbiology and Molecular Genetics, University of the Punjab, Quaid-e-Azam Campus, Lahore-54590, Pakistan \\ *Corresponding author \\ Institute of Microbiology and Molecular Genetics, University of the Punjab, Quaid-e-Azam Campus, Lahore-54590, Pakistan. \\ Email: shabana.mmg@pu.edu.pk
}

\begin{abstract}
Diabetes mellitus is a group of metabolic anomalies due to defect in insulin secretion/action. In type I diabetes, there is complete absence of insulin due to destruction of pancreatic beta cells, whereas type 2 diabetes usually involves insulin resistance. A complex interaction between various environmental, genetic and immunological factors leads to development of diabetes. One major problem for treating T1DM is its difficult prognosis because it leads to many complications, including autoimmune gastritis and celiac diseases. In autoimmune gastritis auto/parietal cell antibodies are formed against the intrinsic factor leading to the gastric atrophy. While in celiac disease, intestinal villi atrophy occurs. Both these conditions have a common clinical outcome which is pernicious anemia. The patients suffering from type I diabetes have approximately threefold increased risk for developing vitamin B12 deficiency as compared to the general population. The major genetic risk factor responsible for this disease is any functional change in HLA class II alleles (HLA DQ, DR and DP). These genes are present on chromosome 6 and are involved in approximately $40 \%$ cases of type I diabetes mellitus. The production of glutamic acid decarboxylase and anti-thyroid peroxidase antibodies have also been reported to be associated with this genotype. The glutamic acid decarboxylase converts glutamic acid to gamma-amino butyric acid producing hydrochloric acid from gastric parietal cells. The antibodies against this antigen can cause achlorhydria that can ultimately lead to vitamin B12 deficiency. Hematologic and neurological disorders, as well as a variety of cardiovascular diseases, are the most common clinical manifestations of deficiency of vitamin B12.
\end{abstract}

Key words: Diabetes, hyperglycemia, autoimmune diseases, gastritis, vitamin B12 deficiency 


\section{Introduction}

Diabetes mellitus is a metabolic condition that causes continuous hyperglycemia due to decreased insulin secretion or action. Depending upon the condition, diabetes can be of following two types; Type 1 diabetes (T1DM) which is an autoimmune disorder caused by destruction of pancreatic beta cell leading to complete absence of insulin (Goldenberg and Punthakee, 2013) and T2DM (Type 2 diabetes) which is due to insulin resistance as well as impaired insulin secretion. Diabetes can also be categorized depending on whether it is caused by a single gene mutation (monogenic diabetes) or by the quantitative action of many small size variations in multible genes (polygenic diabetes).

\section{T1DM and autoimmune disorders}

The type 1 diabetes is caused due to complex interaction of genetic, immune system, and environmental factors. It is an autoimmune condition caused by the loss of beta cells. It has a difficult prognosis because it is also linked to a number of various autoimmune diseases (De Block et al., 2001) as shown in table 1 .

Table1: Autoimmune disorders in type 1 diabetes (De Block et al. 2001).

\begin{tabular}{|l|l|}
\hline \multicolumn{1}{|c|}{ Autoimmune disorder } & Antibodies \\
\hline $\begin{array}{l}\text { Autoimmune gastritis (responsible for vitamin } \\
\text { B12 deficiency) }\end{array}$ & Parietal Cell Antibodies (PCA) \\
\hline Hashimoto's thyroiditis or Graves' disease & $\begin{array}{l}\text { Thyroid Peroxidase (TPO) } \\
\text { Antibodies }\end{array}$ \\
\hline Celiac disease & Endomysial Antibodies (EmA-IgA) \\
\hline Addison's disease & Anti-Adrenal Antibodies (AAA) \\
\hline
\end{tabular}

\section{Pernicious anemia (PA)}

PA is a chronic disease results in insulin deficiency due to the destruction of pancreatic beta cells. The universal occurrence of type I diabetes in offspring varies from 0.1 to 57.6/100,000 (Mosso et al., 2015). PA is a vitamin B12 (cobalamin) deficiency caused by a lack of gastric intrinsic factor, which results in reduced intestinal absorption. Neurologic diseases, megaloblastic anaemia, and glossitis are the most common clinical symptoms of vitamin B12 insufficiency (Banka et al., 2011). In patients with type I diabetes, the risk of organspecific autoimmune illnesses increases double as compared to the general population, with the prevalence of pernicious anaemia hovering around $0.5-4 \%$ in diabetic patients vs $0.12 \%$ in the general population. Thyroid dysfunction is prevalent in $31.4 \%$ of type I diabetes patients, and it is significantly more prevalent in females. Pernicious anaemia is three times more common in people with type I diabetes than in the general population, according to a study in North America (Perros et al., 2000).

Auto and parietal cell antibodies in TIDM patients generated against intrinsic factor types 1 and 2. By preventing vitamin $\mathrm{B} 12$ binding to intrinsic factor, the absorption from the terminal illeum is inhibited via parietal cell antibody (PCA) against intrinsic factor. The formation of these auto antibodies is observed in approximately $70 \%$ patients suffering from pernicious anemia. Furthermore, celiac disease and primary 
autoimmune hypothyroidism are two conditions that have a direct impact on vitamin B12 metabolism in T1DM (Kibirige and Mwebaze 2013a).

\section{Vitamin B12 metabolism}

It is necessary for regular physiological processes, including DNA synthesis and neurological system (Oh and Brown, 2003). Dietary cobalamin is attached to the animal protein called P-protein and bounded form of cobalamin can be obtained through animal foods. Because of the presence of pepsin and hydrochloric acid in the stomach, this complex of cobalamin and P-protein is disrupted and free cobalamin is released in the body. This free cobalamin then attaches to the R-protein, released by salivary glands and not strongly linked with intrinsic factor present in the stomach. Aforementioned composite is transported to the pancreas where it is degraded by pancreatic enzymes releasing thcobalamin, which tightly binds to the intrinsic factor until it reaches the distal ileum. Transcobalamin I (TCI), II (TCII), and III (TCIII) are transported by cubilin (pesent inside the intestinal epithelium). Transcobalamin II is found in all cells and is responsible for cobalamin delivery via endocytosis. Within the cells, cobalamin is then further transformed into adenosylcobalamin and methylcobalamin (Andrès et al., 2004) as shown in Figure 1.

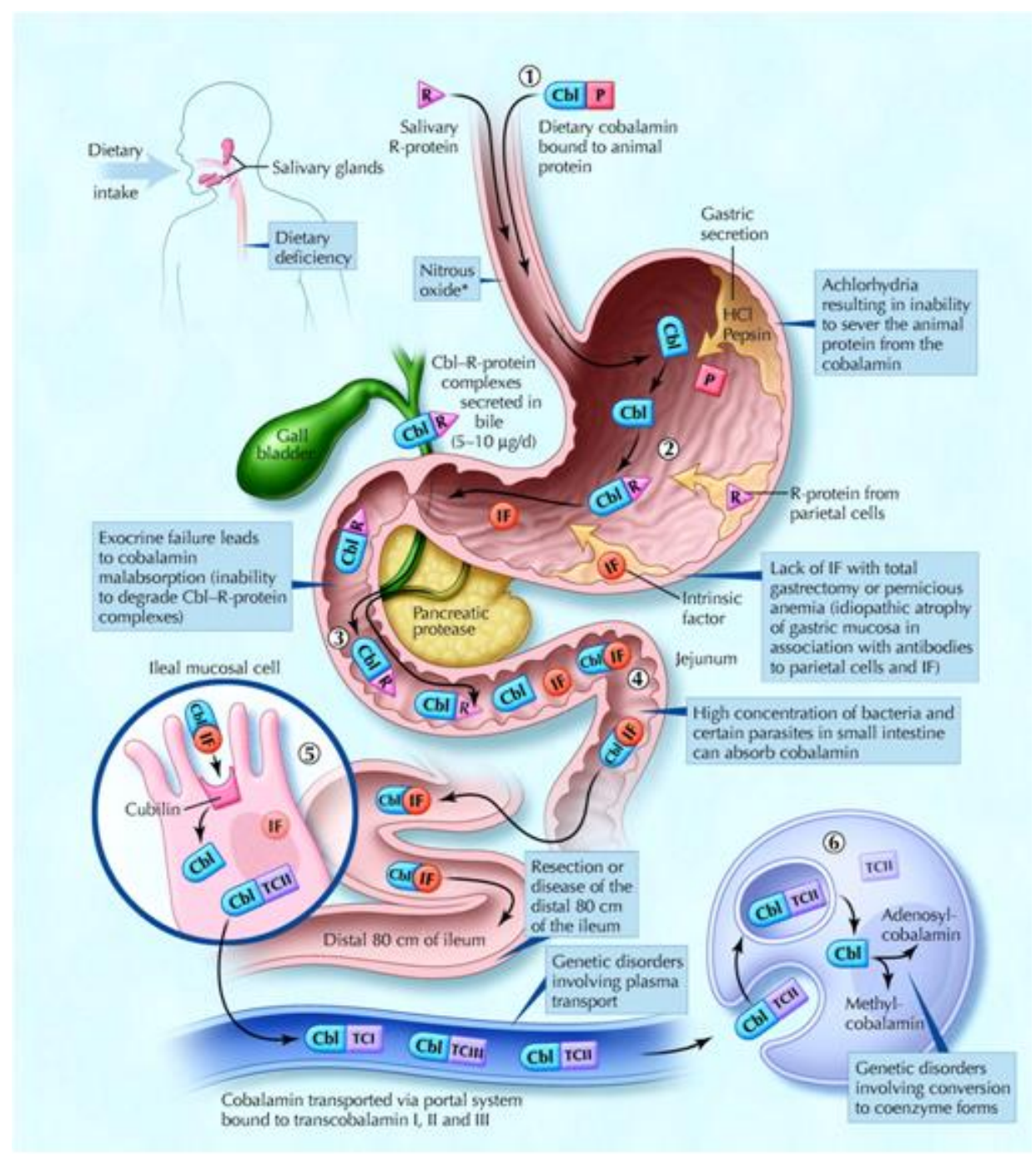

Figure 1: Metabolism of vitamin B12 (Andrès et al., 2004). 


\section{Clinical manifestation of vitamin B12 deficiency}

Most prevalent clinical symptoms are hematologic difficulties (such as megaloblastic anaemia and pancytopenia), neurologic disorders (such as peripheral neuropathy and paresthesias), and cardiovascular diseases (mostly myocardial infarction) (Oh and Brown, 2003).

\section{Vitamin B12 deficiency and Autoimmune gastritis}

In comparison to the general population, where this illness is asymptomatic, young children are more vulnerable to autoimmune gastritis. As the condition progresses, it manifests clinically as an iron shortage or vitamin B12. Gastric cancer can be caused by the atrophic gastritis (Figure 2). HLA haplotypes (HLA-DQA1, HLA-DQB1) are among its genetic reasons. This genotype is also associated with the presence of GAD (glutamic acid decarboxylase) and ATPO (anti-thyroid peroxidase) antibodies. Because most of these loci are found on the same chromosome, type 1 diabetes patients are more likely to develop autoimmune disorders. GAD antigen is the common antigen found in the pancreas and gastric mucosa. This GAD antigen converts glutamic acid to gamma-amino butyric acid (GABA), results in production of hydrochloric acid ( $\mathrm{HCl})$ from gastric parietal cells. The response of T-cells begins in the pancreas and then spreads to other neuroendocrine tissues holding comparable antigens, where activated T cells destroy them (Krzewska and Ben-Skowronek, 2016). Patients of type 1 diabetes have a threefold greater risk of developing pernicious anaemia, as compared to non-diabetics, who have rare cases (prevalence of 0.1-2\%) of pernicious anaemia and atrophic gastritis (Tzellos et al., 2009).

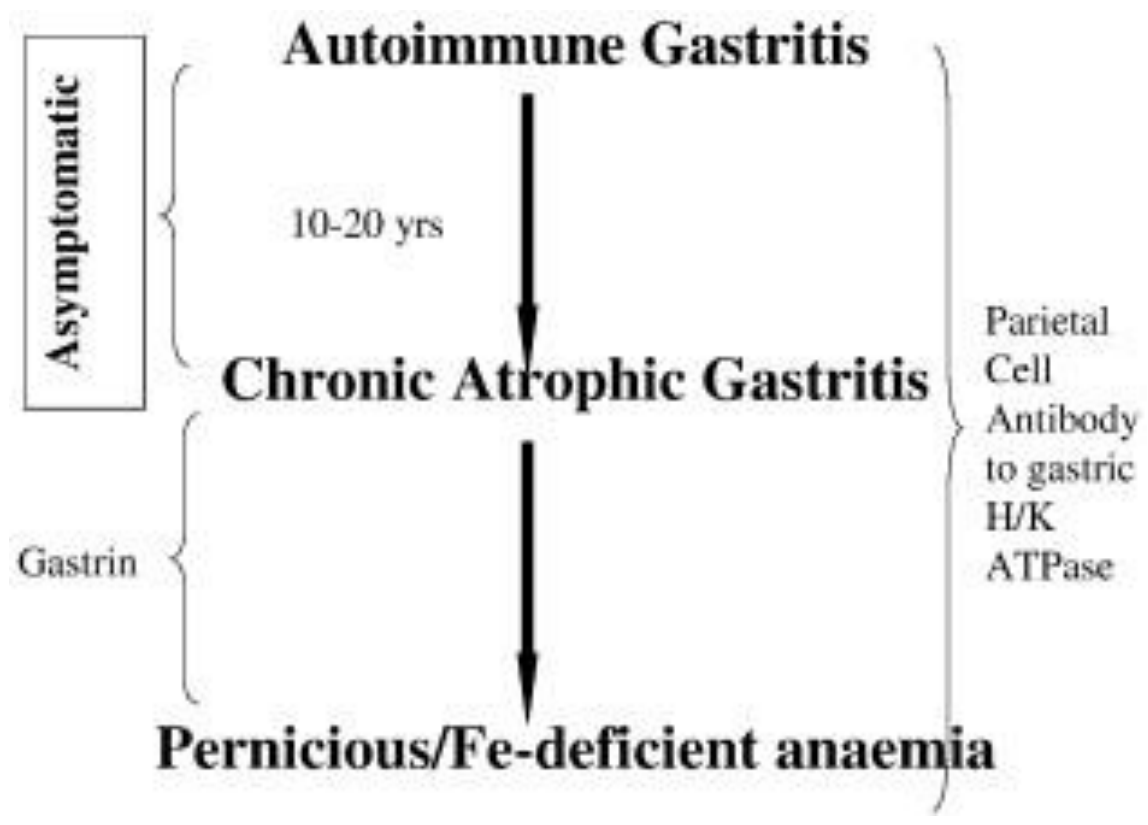

Figure 2: Natural history of autoimmune gastritis and atrophic gastritis (Toh, 2014).

\section{Vitamin B12 deficiency and Celiac disease}

This disease is a multisystemic inflammatory disease of the intestine which is caused by oral intolerance to gluten, wheat, and other grains. In 1969, a link was discovered between type 1 diabetes and celiac disease. Both disorders share a genetic basis in which non-HLA and HLA genes are involved. People with HLA-DQ2/8 positive are more likely to develop celiac disease (Scaramuzza et al., 2013). Celiac disease affects 
1-10\% of diabetic children and adolescents (Kordonouri et al., 2009). The foremost reason of both celiac and type 1 diabetes is the wheat gluten (common environmental factor). Gluten triggers an immune response which results in production of antireticulin (ARA), antiendomysial (EmA), antigliadin (AGA), and anti-tissue transglutaminase (ATG) like auto antibodies. Intestinal villi atrophy can also result from celiac diseases. Due to this there is a high chance that vitamin D, folic acid, fat soluble vitamins, vitamin B12 and calciumwill be less absorbed. Its main clinical signs include growth retardation, bone demineralization, body weight loss, pernicious anaemia and rickets (Krzewska and Ben-Skowronek, 2016).

\section{Identification of Vitamin B12 deficiency in T1DM}

Specific standards for the diagnosis of vitamin B12 deficiency in T1DM patients have not established yet. Because this impairment might arise at any point in T1DM patients, the only option is to check for it at the time of diagnosis and then on and off later (Kibirige and Mwebaze, 2013b). The presence of autoantibodies like PCA or TPA, as well as serum vitamin B12 levels, can be used to screen for deficiency.

\section{Treatment of Vitamin B12 deficiency}

Regardless of whether the patient has type 1 diabetes or type 2 diabetes, replacement therapy, in which patients take oral or parenteral vitamin B12, has been revealed to help patients recover from deficiency. A 100 $\mu \mathrm{g} /$ week oral or intramuscular injection of vitamin B12 is sufficient for juvenile T1DM patients with vitamin B12 insufficiency. However, due to severe deficiency, parenteral or oral treatment of $1000 \mu \mathrm{g} / \mathrm{day}$ upto for one month, followed by one week for each month, is recommended (Andres and Serraj, 2012; De Block et al., 2008).

\section{Conclusion}

Vitamin B12 deficiency is a complication of type I diabetes mellitus, which is caused by a combination of genetic and immunologic causes. Class two human leucocyte antigen, which is located on chromosome 6, is one of the most important genetic risk factors for this disease. Apart from genetic issues, immunologic factors, that cause atrophic gastritis or pernicious anaemia, include parietal cell antibodies that are produced against the intrinsic factor. For the treatment of T1DM and to avoid the progression of linked disorders such as vitamin B12 deficiency, further studies should be conducted to not only determine the optimize dose of vitamin B12 for T1D patients with deficit, but to also identify the risk factors that lead to this insufficiency.

\section{Conflict of Interest}

All Authors declare that they have no conflict of interest.

\section{REFRENCES}

Andrès, E., Loukili, N. H., Noel, E., Kaltenbach, G., Abdelgheni, M. B., Perrin, A. E., Noblet-Dick, M., Maloisel, F., Schlienger, J. L. and Blicklé, J. F. 2004. Vitamin B12 (cobalamin) deficiency in elderly patients. Canadian Medical Association Journal, 171(3):251-259.

Andres, E. and Serraj, K. 2012. Optimal management of pernicious anemia. Journal of Blood Medicine, 3:97. 
The Journal of Microbiology and Molecular Genetics (JMMG)

Vol. 1, No. 2 (2020), Article No. 5, pp. $44-49$

Banka, S., Ryan, K., Thomson, W. and Newman, W. G. 2011. Pernicious anemia-genetic insights. Autoimmunity Reviews, 10:455-459.

De Block, C. E. M., De Leeuw, I. H., Vertommen, J. J. F., Rooman, R. P. A., Du Caju, M. V. L., Van Campenhout, C. M., Weyler, J. J., Winnock, F., Van Autreve, J. and Gorus, F. K. 2001. Beta-cell, thyroid, gastric, adrenal and coeliac autoimmunity and HLA-DQ types in type 1 diabetes. Clinical and Experimental Immunology, 126(2):236-241.

De Block, C. E., De Leeuw, I. H. and Van Gaal, L. F. 2008. Autoimmune gastritis in type 1 diabetes: a clinically oriented review. The Journal of Clinical Endocrinology and Metabolism, 93:363-371.

Goldenberg, R. and Punthakee, Z. 2013. Definition, classification and diagnosis of diabetes, prediabetes and metabolic syndrome. Canadian Journal Of Diabetes, 37:S8-S11.

Kibirige, D. and Mwebaze, R. 2013a. Vitamin B12 deficiency among patients with Diabetes mellitus: is routine screening and supplementation justified? Journal of Diabetes and Metabolic Disorders, 12(1):1-6.

Kibirige, D. and Mwebaze, R. 2013b. Vitamin B12 deficiency among patients with Diabetes mellitus: is routine screening and supplementation justified? Journal of Diabetes and Metabolic Disorders, 12:17 doi:10.1186/2251-6581-12-17.

Kordonouri, O., Maguire, A. M., Knip, M., Schober, E., Lorini, R., Holl, R. W. and Donaghue, K. C. 2009. Other complications and associated conditions with diabetes in children and adolescents. Pediatric Diabetes, 10:204-210.

Krzewska, A. and Ben-Skowronek, I. 2016. Effect of associated autoimmune diseases on Type 1 Diabetes mellitus incidence and metabolic control in children and adolescents. BioMed Research International, 6219730.

Mosso, C., Halabi, V., Ortiz, T. and Hodgson, M. I. 2015. Dietary intake, body composition, and physical activity among young patients with type 1 Diabetes mellitus. Journal of Pediatric Endocrinology and Metabolism, 28:895-902.

Oh, R. and Brown, D. L. 2003. Vitamin B12 deficiency. Americam Family Physician, 67:979-986.

Perros, P., Singh, R., Ludlam, C. and Frier, B. 2000. Prevalence of pernicious anaemia in patients with Type 1 Diabetes mellitus and autoimmune thyroid disease. Diabetic Medicine, 17:749-751.

Scaramuzza, A. E., Mantegazza, C., Bosetti, A. and Zuccotti, G. V. 2013. Type 1 diabetes and celiac disease: The effects of gluten free diet on metabolic control. World Journal of Diabetes, 4:130-134.

Toh, B. H. 2014. Diagnosis and classification of autoimmune gastritis. Autoimmunity Reviews, 13:459-462.

Tzellos, T. G., Tahmatzidis, D. K., Lallas, A., Apostolidou, K., Goulis, D. G. 2009. Pernicious anemia in a patient with Type 1 Diabetes mellitus and alopecia areata universalis. Journal of Diabetes and its Complications, 23:434-437. 